\title{
Use of Vertically-Aligned Carbon Nanotube Array to Enhance the Performance of Electrochemical Capacitors
}

\author{
Mélanie Guittet ${ }^{1}$, Adrianus I. Aria ${ }^{1}$, and Morteza Gharib ${ }^{1}$ \\ ${ }^{1}$ Graduate Aeronautical Laboratories, California Institute of Technology, Pasadena, CA 91125, USA \\ Email: mguittet@caltech.edu, indrat@caltech.edu,mgharib@caltech.edu
}

\begin{abstract}
In the domain of energy storage, electrochemical capacitors have numerous applications ranging from hybrid vehicles to consumer electronics, with very high power density at the cost of relatively low energy storage. Here, we report an approach that uses vertically aligned carbon nanotube arrays as electrodes in electrochemical capacitors. Different electrolytes were used and multiple parameters of carbon nanotube array were compared: carbon nanotube arrays were shown to be two to three times better than graphite in term of specific capacitance, while the surface functionalization was demonstrated to be a critical factor in both aqueous and nonaqueous solutions to increase the specific capacitance. We found that a maximum energy density of 21 Wh/kg at a power density of $1.1 \mathrm{~kW} / \mathrm{kg}$ for a hydrophilic electrode, could be easily achieved by using tetraethylammonium tetrafluoroborate in propylene carbonate. These are encouraging results in the path of energy-storage devices with both high energy density and power density, using only carbon-based materials for the electrodes with a very long lifetime, of tens of thousands of cycles.
\end{abstract}

Index Terms - electrochemical capacitors, carbon nanotubes, functionalization, long lifetime

\section{INTRODUCTION}

The use of carbon nanotubes (CNT) as electrodes in electrochemical double-layer capacitors (EDLC) has proven to be very promising, mainly because of the high surface area, high electrical conductivity and small charge separation. However, vertically-aligned CNT arrays (VANT) has not been intensively researched despite its very promising features, mainly the fact that the specific capacitance is ten times higher than their non-aligned equivalent [1]. Even though EDLC have lower energy density than Li-ion batteries, other interesting characteristics make them very attractive including long operational life of more than 500000 charge-discharge cycles, lower costs per cycle, and fast charging [2][3]. Previously reported studies indicated that the maximum specific capacitance that could be achieved using purified CNT electrodes was around $135 \mathrm{~F} / \mathrm{g}$ [4]. The result presented herein suggests that the charge storage capacity of electrochemical capacitors can be improved even further by employing VANT that have been functionalized beforehand to increase charge transfer to and from the electrolyte.

\section{EXPERIMENTAL METHOD}

VANT samples were grown using chemical vapour deposition (CVD) on a silicon substrate with ethylene and hydrogen as the precursor gasses. These samples were then transferred onto copper foil tape, which is used as the current collector in electrochemical cell.

Electrochemical measurements were carried out in different electrolytes: $1 \mathrm{M}$ sulfuric acid aqueous electrolyte $\left(\mathrm{H}_{2} \mathrm{SO}_{4}\right), 6 \mathrm{M}$ potassium hydroxide aqueous electrolyte $(\mathrm{KOH})$, and a solution of tetraethylammonium tetrafluoroborate $\left(\mathrm{Et}_{4} \mathrm{NBF}_{4}\right)$ in nonaqueous propylene carbonate (PC). Cyclic voltammetry (CV), electrochemical impedance spectroscopy (EIS), as well as charge-discharge tests using galvanostatic cycling were used to assess the specific capacitance of the VANT in different electrolytes. For comparison, the specific capacitance of graphite was also measured. The graphite sheet is composed of two thin layers of graphite, held by a copper film in between.

The effect of surface functionalization in the specific capacitance of the VANT was also studied by comparing the hydrophobic arrays to the hydrophilic counterparts in the abovementioned electrolytes. Here, the hydrophobic CNT array is defined as an array that has purity higher than $95 \%$ and has static contact angle for water higher than $150^{\circ}$. On the other hand, the hydrophilic CNT array is defined as an array that has been previously functionalized with oxygenated functional groups, e.g. hydroxyl groups, via oxidation process and has static contact angle for water lower than $30^{\circ}$. The capability of the array to withstand thousands of charging and discharging cycles was also assessed.

The goal of this study was not to achieve the maximum values, in term of specific capacitance, energy density or power density, but to make a direct comparison between 
Figure a

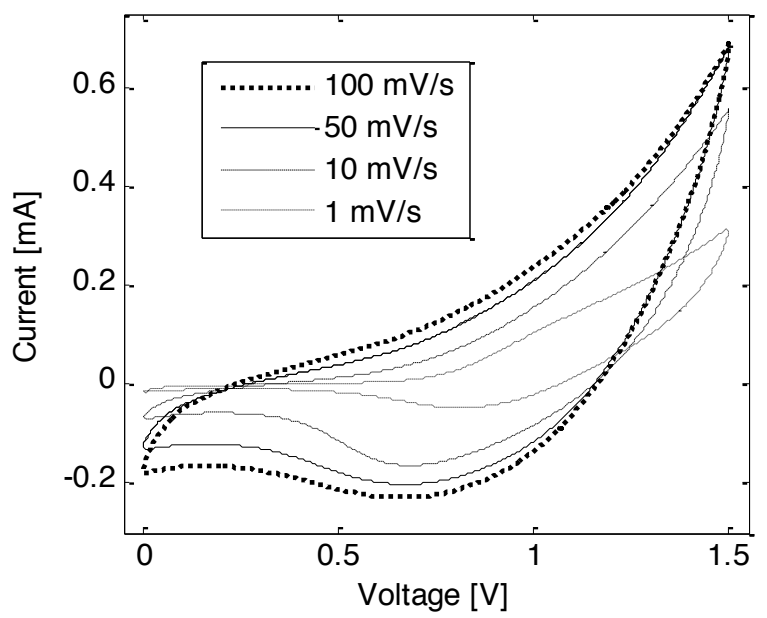

Figure c

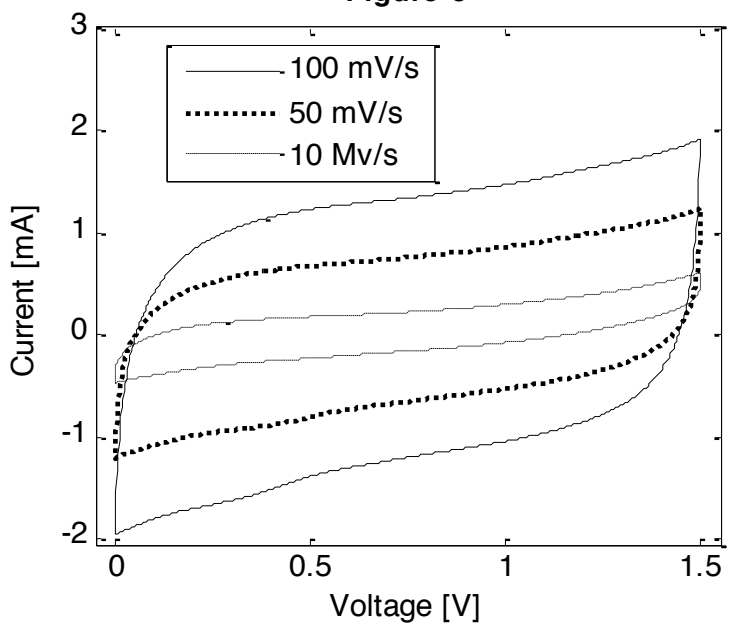

Figure $b$

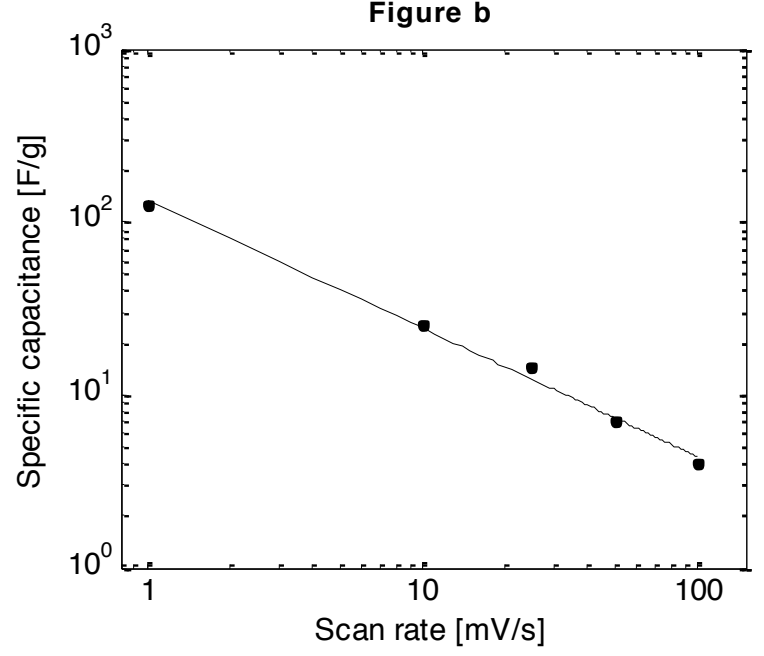

Figure d

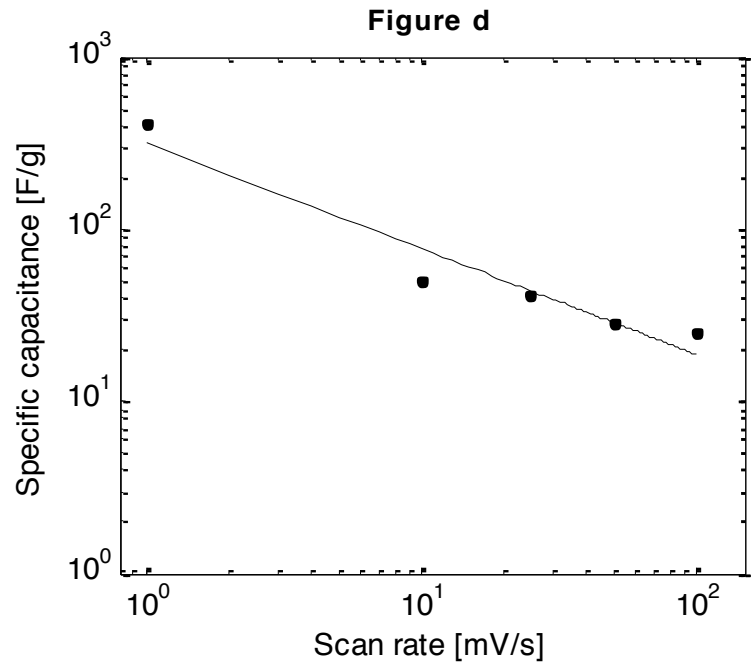

Figure 1| Cyclic voltammograms (a) Symmetric electrodes made of graphite, in a solution of $1 \mathrm{M} \mathrm{Et}_{4} \mathrm{NBF}_{4}-\mathrm{PC}$ for different scan rates. The shape is nearly independent of the scan rates when the scan rate is higher than $25 \mathrm{mV} / \mathrm{s}$ (b) Power relationship between the specific capacitance and the scan rate, for electrodes made of graphite in $1 \mathrm{M} \mathrm{Et}_{4} \mathrm{NBF}_{4}-\mathrm{PC}$ (c) Symmetric electrodes composed of VANT, in $1 \mathrm{M}^{\mathrm{E}} \mathrm{t}_{4} \mathrm{NBF}_{4}-\mathrm{PC}_{\text {for different }}$ scan rates (d) Power relationship between the specific capacitance and the scan rate for VANT, in $1 \mathrm{M} \mathrm{Et}_{4} \mathrm{NBF}_{4}-\mathrm{PC}$

graphite-based electrodes and VANT-based electrode, as well as to assess the effect of functionalization on the overall performance. The electrode lifetime was thoroughly investigated, as a crucial part of this research.

\section{RESULTS AND DISCUSSION}

\section{A. Capacitive behavior}

The capacitive behavior of a double-layer capacitor fabricated using both graphite- and VANT-based electrodes was measured, as seen on fig. 1. Cyclic voltammograms were recorded at various scan rates, ranging from $1 \mathrm{mV} / \mathrm{s}$ to
$100 \mathrm{mV} / \mathrm{s}$. As expected, the cyclic voltammogram of the VANT fig. 1c shows a good rectangular and symmetric shape over a large range of scan rates. The charge and discharge processes are very fast at the VANT-electrolyte interface, and the voltammogram is perfectly featureless, confirming the usefulness of VANT as electrodes for supercapacitors. It is clear from fig.1c that the voltammogram of the VANT is highly dependent on the voltage scan rate. In fact, it is rare to find a voltammogram that is independent of the voltage scan rate [5]. This voltammogram also indicates some losses due to internal resistance, which may be caused by the high concentration of the electrolyte. At a considerably high concentration, the electrolyte cannot be fully ionized, such that the current is 

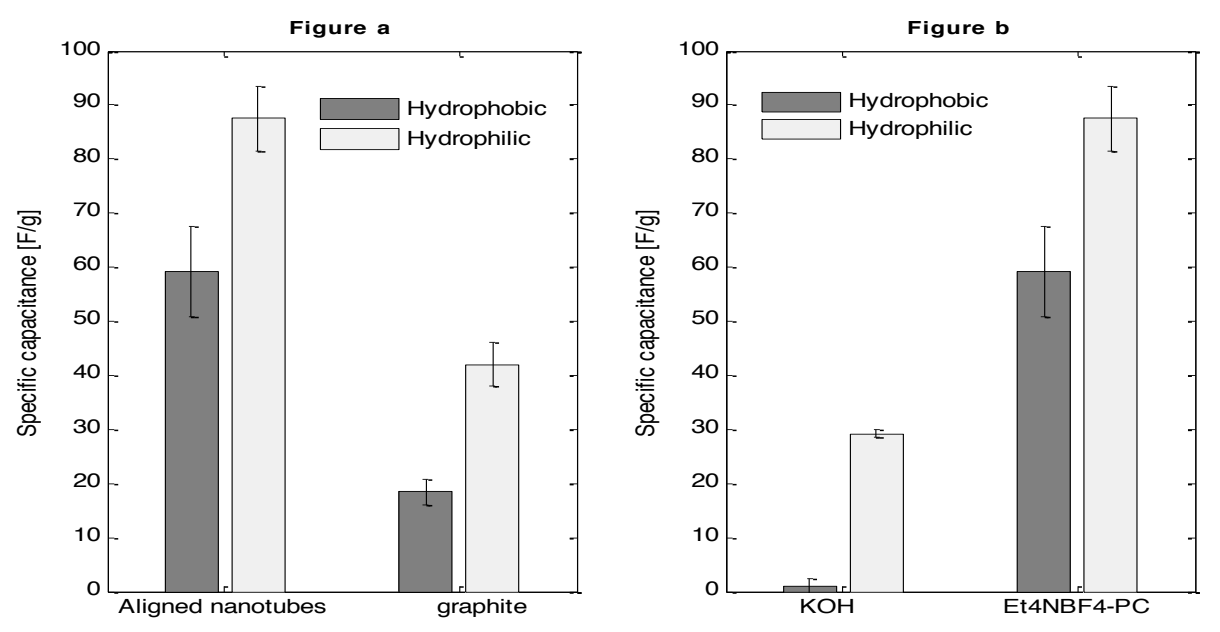

Figure 2 | Surface functionalization (a) Specific capacitance of the vertically-aligned CNT array electrodes in $1 \mathrm{M} \mathrm{Et}_{4} \mathrm{NBF}_{4}-\mathrm{PC}$ is extremely higher than that of the graphite, in both cases of hydrophilic and hydrophobic, at $25 \mathrm{mV} / \mathrm{s}$. (b) Specific capacitance of the vertically-aligned CNT array electrodes in both aqueous $(6 \mathrm{M} \mathrm{KOH})$ and non-aqueous $\left(1 \mathrm{M} \mathrm{Et}_{4} \mathrm{NBF}_{4}-\mathrm{PC}\right)$ solution, measured at $25 \mathrm{mV} / \mathrm{s}$.

not carried at its maximum efficiency. Nevertheless, a concentrated electrolyte allows for an increase in the specific capacitance. Low contact resistance and good capacitive behaviour can be assumed since no significant difference appeared between bare samples and samples with metal contact. It is important to highlight the behavior of the specific capacitance as a function of the scan rate follows a power law, which suggests that the process is not diffusioncontrolled [6].

In contrast, the graphite voltammogram shows narrow loops with a large oblique angle and an elbow around $0.8 \mathrm{~V}$ (fig 1a), which is typical of a highly resistive electrode [7]. A large non-capacitative current response is also present between 0.8 and $1.5 \mathrm{~V}$ for the graphite sample (fig 1a), which is quite unexpected due to the relatively high conductivity of graphite. The shape clearly shows that the graphite-based electrode cannot be used at a voltage range of $0-1.5 \mathrm{~V}$. A much better voltammograms were obtained if the voltage range is limited to $0-1 \mathrm{~V}$. Interestingly, the voltammogram of the graphite-based electrode is nearly independent of scan rate for scan rates over $50 \mathrm{mV} / \mathrm{s}$. This mean the size of the diffusion layer is more or less constant for the scan rates higher than $50 \mathrm{mV} / \mathrm{s}$. We can suppose this is the minimum diffusion layer size possible since slow scan rates have larger diffusion layers.

These lower values of specific capacitance for graphite are the result of the lower surface area available since only the top layer of graphite is accessible, and can participate as the current increases. On the contrary, VANT offer a very large surface area, and thus gives higher specific capacitance. These results are in accordance with previous studies [1].

\section{B. Aligned nanotubes vs graphite}

VANT electrodes were compared to graphite electrodes to show that the former would yield higher specific capacitance. A $1 \mathrm{M} \mathrm{Et}_{4} \mathrm{NBF}_{4}-\mathrm{PC}$ solution was used to make the comparison, and the result was then compared to literature. Specific capacitance of the VANT electrodes in $1 \mathrm{M} \mathrm{Et}_{4} \mathrm{NBF}_{4}-\mathrm{PC}$ is about two to three times higher than that obtained with graphite electrodes, with a value of $20 \mathrm{~F} / \mathrm{g}$ for the hydrophobic graphite electrode against $60 \mathrm{~F} / \mathrm{g}$ for hydrophobic VANT electrode. We also found an increase from $42 \mathrm{~F} / \mathrm{g}$ for a hydrophilic graphite electrode to nearly 90 $\mathrm{F} / \mathrm{g}$ for a hydrophilic VANT array electrode, as seen on fig 2a. These findings agree with the recent reported study [1] confirming the importance of structurally aligning nanotubes in the direction of ion diffusion to increase the specific capacity. This leads to both an increase of the surface area available because the ions can access more easily and freely the core of the nanotubes, and a diminution in the overall resistance of the system. This hypothesis is confirmed by the electrochemical impedance spectroscopy (EIS) experiments, where the resistance of graphite-based electrodes is about $\sim 10 \mathrm{k} \Omega \pm 0.3 \mathrm{k} \Omega$, while the resistance of VANT-based electrodes is about $\sim 1.5 \mathrm{k} \Omega \pm 0.1 \mathrm{k} \Omega$, which is nearly an order of magnitude lower.

Graphite, as an allotrope of carbon with sp2 hybridization, shares a lot of common properties with carbon nanotubes, including good electrical conductivity. However, the specific capacitance obtained when graphite is used as the electrode yields considerably lower specific capacitance, namely 3 to 5 times lower for the same scan rate than what can be achieved with VANT, as seen in fig $1 \mathbf{b}$ and fig 1d. A power law relationship can be deduced from these figures. he specific capacitance $(S C$, in $\mathrm{F} / \mathrm{g}$ ) is linked to the scan rate ( $v$, in $\mathrm{mV} / \mathrm{s}$ ) via the formula: 

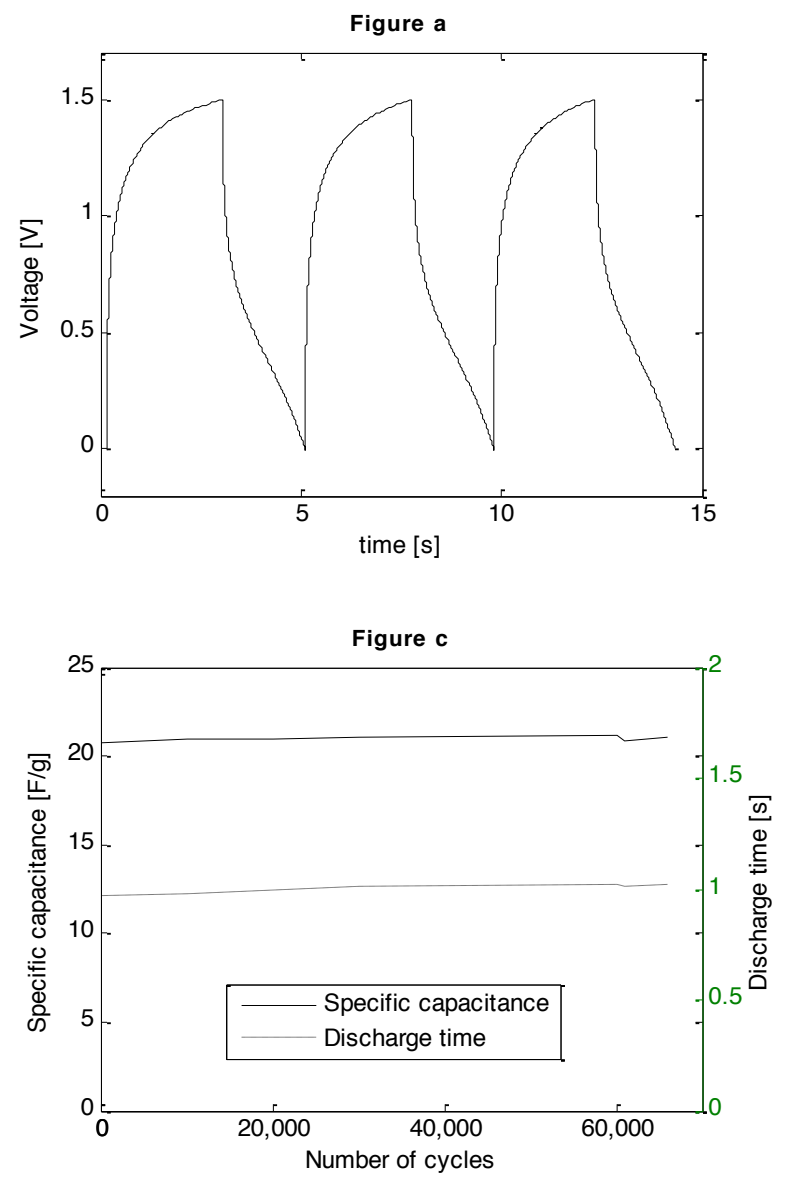
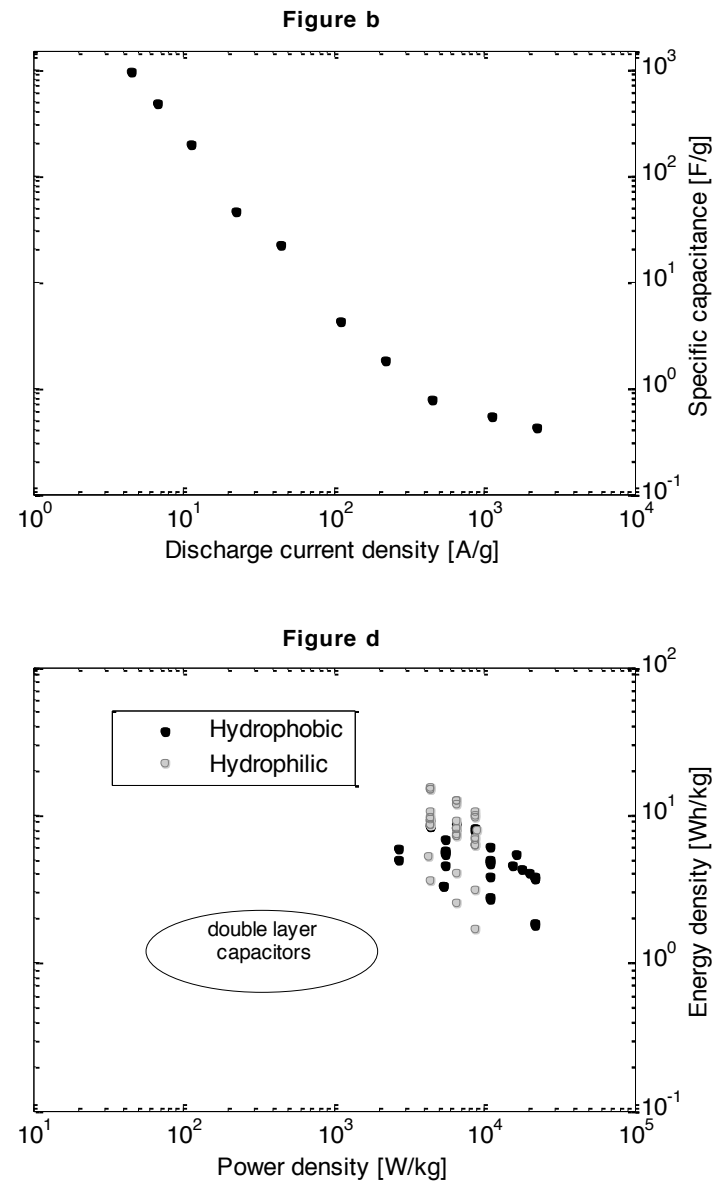

Figure 3 | Electrochemical performance. (a) Galvanostatic charge-discharge at constant current, zeroed in time (b) Specific capacitance vs current density. Under the hypothesis that capacitance is constant over time, the specific capacitance SC was calculated from the discharge curves according to $S C=\frac{I(\Delta t)^{2}}{2 \int_{0}^{T} V d t}$, where I is the discharge current, $\Delta t$ the discharge time, and $\int_{0}^{T} V d t_{\text {the area under the }}$ discharge curve.

(c) Specific capacitance in function of cycles, in Et4NBF4-PC, $>70000$ cycles, 100mV/s (d) Ragone plot (considering only active material)

$$
S C \approx \alpha\left(\frac{d V}{d t}\right)
$$

Where $\alpha$ and $\beta$ for the graphite-based electrode are found to be 130 and $-3 / 4$ respectively. In the case of VANT-based electrode, $\alpha$ and $\beta$ are found to be 310 and $-3 / 5$ respectively.

\section{Role of functionalization: Hydrophilic vs hydrophobic}

In agreement to some previously reported studies [9][10], the specific capacitance of the VANT electrode in aqueous solutions can be increased significantly by the addition of hydroxyl and carboxyl groups to the electrode. The presence of such surface functional groups increases the number of contact sites between the electrode and the electrolyte molecules, which in turn increases the overall charge transfer between the electrode and electrolyte. Therefore, a hydrophilic VANT sample is expected to give higher specific capacitance in polar protic electrolyte, because it allows a better charge transfer between the electrolyte and the VANT electrode compared to the hydrophobic one.

In the case of VANT electrodes in $1 \mathrm{M} \mathrm{H}_{2} \mathrm{SO}_{4}$ solution, the specific capacitance increases from $16 \mathrm{~F} / \mathrm{g}$ to $51 \mathrm{~F} / \mathrm{g}$ once the VANT electrodes are hydrophilic. Similar behavior was also observed in $6 \mathrm{M} \mathrm{KOH}$, where the specific capacitance increases from $1 \mathrm{~F} / \mathrm{g}$ to nearly $30 \mathrm{~F} / \mathrm{g}$ once they are functionalized, as shown on fig. 2b. Indeed, surface modification by the oxygenated functional groups is supposed to increase the performance by reducing the voltage difference during charge and discharge [9].

When using a polar aprotic solution as the electrolyte the results are similar. PC is an aprotic solvent, which cannot donate protons, so there is no possible hydrogen interaction with the hydroxyl or carboxyl functional groups that were attached to the VANT. However, the presence of those functional groups increases the polarity of the VANT, such 
that dipole-dipole interactions between PC and VANT become more pronounced. In fact, the specific capacitance of the hydrophilic electrodes in $1 \mathrm{M} \mathrm{Et}_{4} \mathrm{NBF}_{4}-\mathrm{PC}$ was measured to be as high as $90 \mathrm{~F} / \mathrm{g}$, which is about $50 \%$ higher than that of the hydrophobic ones, which was measured to be around $60 \mathrm{~F} / \mathrm{g}$ (fig. 2b).

Indeed, PC and water are both polar, and even though the first is aprotic while the second is protic, in both cases an increase in hydrophilicity leads to an increase in specific capacitance.

These results are encouraging compared to recent findings: VANT arrays electrode gives a specific capacitance of $83 \mathrm{~F} / \mathrm{g}$ in the same $1 \mathrm{M} \mathrm{Et}_{4} \mathrm{NBF}_{4}-\mathrm{PC}$ electrolyte [8], which is about what we find in this current study when using the hydrophilic CNT array electrodes $(88 \mathrm{~F} / \mathrm{g}$, as seen on fig $\mathbf{2 b}$ ). The number is lower though than other recent findings with different electrolytes, which can achieve up to $135 \mathrm{~F} / \mathrm{g}$ [4], using either $\mathrm{LiBF}_{4}$ in ethylene carbonate/diethyl carbonate as the electrolyte, and electrochemical lithium insertion into the nanotubular carbon host, or $6 \mathrm{M} \mathrm{KOH}$.

\section{Energy density, power density, and lifetime}

The charge-discharge curves are presented on fig. 3a, for the first three cycles. The non-linear and non-triangular shape is common with dissipation and ohmic drops. The specific capacitance in function of the discharge current is shown on fig. $\mathbf{3 b}$. The maximum specific capacitance was found to be $\sim 1 \mathrm{kF} / \mathrm{g}$ at a discharge current density of $4 \mathrm{~A} / \mathrm{g}$, if we consider only the active material. The specific capacitance decreases as the discharge current is increased as reported by previous studies [11], from $0.95 \mathrm{kF} / \mathrm{g}$ to $0.4 \mathrm{~F} / \mathrm{g}$ as the current discharge increases from $4.5 \mathrm{~A} / \mathrm{g}$ to $2.5 \mathrm{kA} / \mathrm{g}$. This can mainly be explained by the lower mobility due to diffusion of the ions [1]. One can highlight that the specific capacitance decreases by about two orders of magnitude when the discharge current is increased by only one order of magnitude. When the discharge current is low enough, meaning below $\sim 1000 \mathrm{~A} / \mathrm{g}$, the relation between the specific capacitance and the discharge current follows a power law. A comparable number of nearly $1000 \mathrm{~F} / \mathrm{g}$ can be found with cyclic voltammetry with a very low scan rate (around 0.15 $\mathrm{mV} / \mathrm{s}$ ) assuming the relation (2) holds for low scan rate as well. Such a slow regime is yet to be explored. When taking into account the overall weight of the device including the filter and current collectors, the maximum specific capacitance achieved is $0.8 \mathrm{~F} / \mathrm{g}$ for a discharge current of 3.5 $\mathrm{mA} / \mathrm{g}$.

Using the results from the $1 \mathrm{M} \mathrm{Et}_{4} \mathrm{NBF}_{4}$ experiments, the maximum energy density was found to be $21 \mathrm{Wh} / \mathrm{kg}$ at power density of $\sim 1.1 \mathrm{~kW} / \mathrm{kg}$ for a hydrophilic electrode, and the maximum power density was found to be $\sim 22 \mathrm{~kW} / \mathrm{kg}$ at energy density of $\sim 2 \mathrm{Wh} / \mathrm{kg}$ for a hydrophobic sample as seen on fig. 3d. It is interesting to highlight the different behavior whether or not the VANT array electrode is functionalized. When hydrophilic, the specific capacitance is higher, as already shown in fig $\mathbf{2}$, and as a result, the energy density of the hydrophilic samples is twice that of the hydrophobic electrodes. On the contrary, the power density is higher by a factor two when using hydrophobic VANT array electrodes. PC is here used as a polar solvent [12], so the interaction between the non-polar hydrophobic surface of the VANT array and the polar electrolyte generates a very strong repulsion. When a current is applied, the induced repulsion enhances the charge transport, and hence the power is increased. In the other case, when hydrophilic CNT arrays electrode are in contact with polar electrolyte, dipole-dipole interactions are created between the ions and the nanotubes, which must then be broken, resulting in a lower charge flow and thus lower power. These values of power density and energy density were calculated using only the weight of the active material, i.e., the VANT. However, if we take into account the weight of the overall components including current collector and filter, we obtain a maximum energy density of $0.17 \mathrm{Wh} / \mathrm{kg}$ at power density of $9 \mathrm{~W} / \mathrm{kg}$, and a maximum power density of $175 \mathrm{~W} / \mathrm{kg}$ at energy density of $15 \mathrm{mWh} / \mathrm{kg}$. Even though these numbers are not attractive, one has to remember that copper is used as a current collector. Copper is extremely heavy, about two orders of magnitude heavier than the VANT. This major drawback could be overcome by using other lighter weight current collectors, as already proposed by numerous other groups facing the same issue [13].

The lifetime is extremely long, with close to a hundred thousands of cycles, with nearly no change in specific capacitance. The first fifty thousand cycles are shown on fig 3c, showing no change in the specific capacitance as well as in the discharge time. A couple of cycles were needed to reach the maximum value of specific capacitance. The experiment was stopped after $\sim 70000$ cycles, not because of the degradation of the electrodes but because of time consideration. More extended work considering full lifetime will be conducted in the future. These findings are extremely encouraging, although the energy density is smaller in comparison to some earlier reported studies using nanotubes and silver nanowire films on a highly conductive paper, in a lithium-based electrolyte $\left(\mathrm{LiPF}_{6}\right.$ in ethylene carbonate). [14]. However, these quality materials are extremely expensive and present some inherent danger due to their reactive nature. The working voltage range chosen here is 0 $1.5 \mathrm{~V}$, which allows for a longer lifetime at the cost of a lower energy density.

Even though the performance obtained are lower than what can be currently achieved using lithium-based electrolytes [14], specific capacitance of $\sim 135 \mathrm{~F} / \mathrm{g}$ for CNTbased electrodes [4]), this system still harvests a lot of advantages that skirt these lower values. First of all because the carbon nanotubes, as well as the copper and polypropylene filter, are cheap or expected to be much cheaper in the near future. Moreover, the small ecological print of this device should be highlighted, indeed, most batteries use an electrolyte derived from lithium, which is 
not environmentally sound. According to the latest report of Meridian International Research, the mass production of lithium will cause great damages to endangered ecosystems [15]. Moreover, lithium-based batteries will almost entirely be dedicated to portable electronics, not towards large-scale batteries for transportation or industrial use because the production can only sustain the first market [15]. We thus need to find other materials for such large scale batteries. This system is also not dangerous, since it does not carry the set of reactivity issues involved with lithium. The danger of a leak, leading to an explosion is not an issue with lithium-free carbon based EDLCs.

Finally, the potential range chosen does not allow tremendous energy and power densities, but it allows a lifetime of potentially more than hundreds of thousands of cycles. In comparison, most articles report a potential range from 0 to $3 \mathrm{~V}$ [14], but the lifetime is only some hundreds of cycles.

\section{ACKNOWLEDGMENT}

This work was supported by The Charyk Foundation and The Fletcher Jones Foundation under grant number 9900600 and made use of facilities managed by Kavli Nanoscience Institute at the California Institute of Technology.

\section{REFERENCES}

[1] D. T. Welna, L. Qu, B. E. Taylor, L. Dai and M. F. Durstock, "Vertically aligned carbon nanotube electrodes for lithium-ion batteries", Journal of Power Sources, Volume 196, Issue 3, p. 1455$1460,(2011)$
[2] P. Simon and Y. Gogotsi, "Materials for electrochemical capacitors", Nat. Mat, 7, p. 845-854, (2008)

[3] B. E. Conway, "Electrochemical supercapacitors: Scientific Fundamentals and Technological Applications", Kluwer Academics/Plenum Publishers: New York, Voll, p.698, (1999)

[4] E. Frackowiak, et al., "Supercapacitor electrodes from multiwalled carbon nanotubes", Applied Physics Letters, Volume 77, Issue 15 (2000)

[5] Francesco Lufrano and Pietro Staiti, "Conductivity and Capacitance Properties of a Supercapacitor Based on Nafion Electrolyte in a Nonaqueous System", Electrochemical and Solid-State Letters, Volume7, Issue 11, p. A447-A450, (2004)

[6] J.H. Chen, W.Z. Li, D.Z. Wang, S.X. Yang, J.G. Wen, Z.F. Ren, "Electrochemical characterization of carbon nanotubes as electrode in electrochemical double-layer capacitors", Carbon, Volume 40, p. 11931197, (2002)

[7] Chunsheng Du, Ning Pan, "Supercapacitors using carbon nanotubes films by electrophoretic deposition", Journal of Power Sources, Volume 160, p.1487-1494, (2006)

[8] Takayuki Iwasaki and al., "Highly selective growth of vertically aligned double-walled carbon nanotubes by a controlled heating method and their electric double-layer capacitor properties", Physica status Solidi (RRL) Volume 2, No. 2, p. 53-55 (2008)

[9] Seung Woo Lee and al., "High power Li batteries from functionalized CNT electrodes", Nature Nanotechnology, Volume 5, p. 531-537, (2010)

[10]Andreas Hirsch, "Functionalization of Single-Walled Carbon Nanotubes", Angewandte Chemie International. Edition, Volume 41, No. 11 (2002)

[11]Jingxing Chenand al., "Mesoporous Carbon Spheres: Synthesis, Characterization and Supercapacitance", Int. J. Electrochem. Sci., Volume 4, p.1063 - 1073 (2009)

[12]Patent Sherman, B.C.: US20006159933 (2000).

[13]X. Lang, A. Hirata, T. Fujita, and M. Chen, "Nanoporous metal/oxide hybrid electrodes for electrochemical supercapacitors", Nature Nanotechnology, (2011)

[14]L. Hu, J. W. Choi, Y. Yang, S. Jeong, F. La Mantia, L. Cui and Y. Cui, "Highly conductive paper for energy-storage devices", PNAS, Volume 106, Issue 51, p. 21490-21494 (2009)

[15] Meridian International Research, Report: The trouble with lithium2 